\title{
A Hybrid Algorithm for Coverage Path Planning With Imperfect Sensors
}

\author{
Michael Morin ${ }^{1, \bigotimes}$
}

\author{
Irène Abi-Zeid ${ }^{2}$
}

\author{
Yvan Petillot ${ }^{3}$
}

\author{
Claude-Guy Quimper ${ }^{1}$
}

given minimal coverage (minimal conditional probability of detection) must be achieved over the whole area of interest for the path to be feasible.

$C P P$ problems are often solved in order to plan an agent's (or multiple agents) path in such a way to guarantee complete coverage [6], or in the case of imperfect sensors, a minimal required coverage [7]. In complete $C P P$ problems with perfect sensors, a cell is fully covered after a single scan and no further visits are needed. Complete $C P P$ problems (also known as area covering, or region filling) arise in many practical applications, e.g., minesweeping [8], [9], seabed surveys in harbors and waterways [2], robotic mowing, harvesting and ploughing in agricultural applications [10], marine habitat planning [11], and floor cleaning [12].

A $C P P$ problem is different from classical path planning problems where the robot moves from an initial point to a known destination. It is also different from optimal detection search problems for search and rescue or surveillance [13], [14]. In detection search theory, the goal is often to plan a path that maximizes the global probability of finding a search object (e.g., survivor, crashed plane, lost vessel). In these problems, the search stops after detection and the probability distribution of the whereabouts of the search object is often known a priori. In most $C P P$ problems, the objective is to attain a complete coverage of the whole environment. Furthermore, in $C P P$, the whereabouts of objects locations are often unknown, although some authors assume a priori knowledge of targets locations [15], [16], [17].

Time to completion is an important issue. Longer paths to cover an area take more time. Turning also takes more time and may increase navigational errors [18]. Therefore, the goal is to find a feasible path that minimizes the total traveled distance and the total number of turns.

This paper is organized as follows. We discuss related work in section II. Section III formalizes our problem in the context of underwater minesweeping operations. Section IV describes our novel hybrid algorithm based on dynamic programming and on a traveling salesman problem (TSP) reduction. Section V presents, compares and discusses our experimental results. We conclude in section VI.

\section{RELATED WORK}

*This work was partially funded by the Natural Sciences and Engineering
Research Council of Canada and by the Fonds de recherche du QuébecNature et technologies.

${ }^{1}$ M. Morin and C.G. Quimper are with the Department of Computer Science and Software Engineering, Université Laval, Québec, Qc, Canada ${ }^{\otimes}$ Michael.Morin. 3 at ulaval.ca

${ }^{2} \mathrm{I}$. Abi-Zeid is with the Department of Operations and Decision Systems, Université Laval, Québec, Qc, Canada

${ }^{3}$ Y. Petillot is with the Ocean Systems Laboratory, Heriot-Watt University, Edinburgh, Scotland, UK
Most path planning algorithms use a discretized representation of the continuous environment. In a cellular decomposition, the continuous environment is divided in a set of uniform or non-uniform cells [19]. Uniform cellular decompositions involve grids where cells have the same size. If the environment contains obstacles, we have an occupancy 
grid [20]. Such a decomposition technique is considered to be approximate since some cells may be partially obstructed and some parts of the environment may not be fully covered.

In a non-uniform cellular decomposition the constraint on the cell size is relaxed. These include trapezoidal decomposition, boustrophedon decomposition (both exact), and recursive cellular decomposition (approximate). In a $C P P$, when the cellular decomposition results in cells that are larger than the range of the sensor, a lawnmower pattern is often assumed in the cells [18]. Depending on the discretization scale and on the range of the sensor, whether limited to its circumference, extended, or infinite, distant cells may or may not be surveyed. When the environment is known a priori, the problem may be formulated as an off-line CPP problem. Otherwise, we have an on-line $C P P$ problem where the agent must discover its environment. For on-line $C P P$ problems a sensor-based approach [18] is used, i.e., the robot uses its sensors to acquire knowledge on its environment. In our case, since the seabed map is available as a grid of uniform square cells, we have an off-line $C P P$ problem where the discretization scale is such that the side of a cell is not larger than the sonar's range.

There exists a large body of literature on path planning in robotics, mostly for ground robotics in four general areas: navigation, coverage, localization and mapping. $C P P$ methods and path planning for navigation are reviewed in [18] and [21] respectively.

The problem presented in this paper differs from classical $C P P$ problems in three ways: First, the sensors are imperfect; and second, the range of detection is not limited to the location of the AUV and varies with the distance separating it from the scanned area, and with the seabed type. Third, we do not require perfect coverage, we rather must ensure a minimum coverage level everywhere in the area of interest. We call this formulation, the CPP with imperfect extended detection (CPPIED). To our knowledge, the only published work on this formulation is that of [7]. He presented the advantages and disadvantages of existing approaches and concluded that none of them was suitable for the CPPIED. He proposed the heterogeneous coverage path planning (HCPP) algorithm for planning an AUV's path, a highly instance dependent heuristic that must be fine-tuned in a trial and error fashion.

As the CPPIED problem deals with a required coverage in all cells, we also need to distinguish it from the multirobotcontrolled frequency coverage ( $M R C F C$ ) problem [22]. The two problems are similar in that many visits will be needed in each location (cell). However, the goal of the former is to achieve the required coverage in each cell whereas the goal of the latter is to be as close as possible to the prescribed relative frequency of visits.

\section{The CPPIED PRoblem}

Let $\mathcal{T}$ be the set of seabed types, for example, flat or ripple sand. The ocean is represented by a $m \times n$ matrix $\mathbf{O}$ such that $\mathbf{O}_{i j} \in \mathcal{T}$ is the seabed type in the cell $(i, j)$. The cell $(1,1)$ is located in the upper left corner of the grid. The

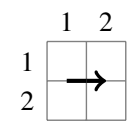

(a) A move from cell $(1,1)$ to $(1,2)$

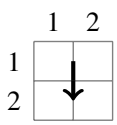

(b) A move from cell $(1,1)$ to $(2,1)$

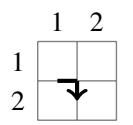

(c) A right turn from cell $(1,1)$ to $(2,1)$

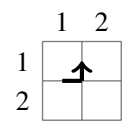

(d) A left turn from cell $(1,1)$ to $(1,1)$
Fig. 1. Feasible moves on a uniform grid

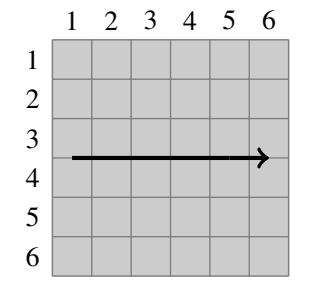

(a) A path from cell $(3,1)$ to cell $(3,6)$ with $r=3$

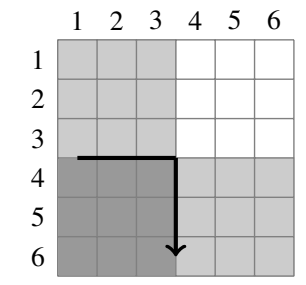

(b) A path from cell $(3,1)$ to cell $(6,3)$ with $r=3$
Fig. 2. Scans on a uniform seabed; light gray shaded cells are scanned once, and dark gray shaded cells are scanned twice.

position of the robot is defined by $((i, j), \operatorname{dir})$ where $(i, j)$ is a grid cell and dir $\in$ \{north, south, east, west $\}$ is the direction in which the robot is pointing. The robot moves on the grid lines between the cells. A robot pointing north or south in cell $(i, j)$ is located in the middle of the vertical line between cells $(i, j)$ and $(i, j+1)$. A robot pointing east or west in cell $(i, j)$ is located in the middle of the horizontal line between cells $(i, j)$ and $(i+1, j)$. The robot moves forward one cell at a time (Fig. 1a and 1b). It cannot stop, backup, nor turn around on the spot. However, $90^{\circ}$ turns while moving forward are allowed (Fig. 1c and 1d). The robot scans $2 r$ cells: $r$ on its left, $r$ on its right. No diagonal scanning occurs while the robot is turning. However, some cells may be scanned a second time just after the turn. Two possible paths and their set of scanned cells are shown on Fig. 2 for range $r=3$.

The conditional detection probability of a sensor scan in a cell $(i, j)$ (given that a mine is present) is a function $p^{\text {scan }}: \mathbb{N}^{+} \times \mathcal{T} \rightarrow[0.0,1.0]$ of the distance $d(x, y, i, j)$, in number of cells, between the current robot's cell $(x, y)$ and the scanned cell $(i, j)$, and of the seabed type $\mathbf{O}_{i j}$ of the scanned cell $(i, j)$. For instance, with $\mathcal{T}=\{$ flat (f), ripples (r), complex (c) $\}$ and $r=3$, the sensor's conditional detection probabilities are expressed as:

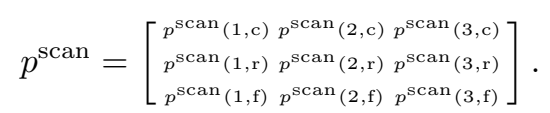

The current coverage map of a grid environment is represented by a $m \times n$ matrix $\mathbf{C}$ where $\mathbf{C}_{i j}$ is the achieved conditional detection probability in the cell $(i, j)$. The initial coverage map is the null matrix. After a first scan of cell $(i, j)$ from cell $(x, y)$, the coverage in $(i, j)$ is $\mathbf{C}_{i j}=p^{\text {scan }}\left(d(x, y, i, j), \mathbf{O}_{i j}\right)$. The conditional probability of non-detection (i.e., the miss probability, or the noncoverage $)$ is $1-\mathbf{C}_{i j}=1-p^{\text {scan }}\left(d(x, y, i, j), \mathbf{O}_{i j}\right)$. Given independent detections and following a scan in cell $(i, j)$ 
from cell $\left(x^{\prime}, y^{\prime}\right)$, the non-coverage in cell $(i, j)$ is $1-$ $\mathbf{C}_{i j}^{\prime}=\left(1-\mathbf{C}_{i j}\right)\left(1-p^{\mathrm{scan}}\left(d\left(x^{\prime}, y^{\prime}, i, j\right)\right), \mathbf{O}_{i j}\right)$. Therefore, the updated coverage of cell $(i, j)$ is $\mathbf{C}_{i j}^{\prime}=1-\left(1-\mathbf{C}_{i j}\right)(1-$ $\left.p^{\text {scan }}\left(d\left(x^{\prime}, y^{\prime}, i, j\right)\right), \mathbf{O}_{i j}\right)$ leading to the update equation (2).

$$
\mathbf{C}_{i j}^{\prime}:=\mathbf{C}_{i j}+\left(1-\mathbf{C}_{i j}\right) p^{\mathrm{scan}}\left(d\left(x^{\prime}, y^{\prime}, i, j\right), \mathbf{O}_{i j}\right) .
$$

The required coverage is represented by a $m \times n$ matrix $\mathbf{D}$ such that $\mathbf{D}_{i j}$ represents the required minimum coverage that must be attained in cell $(i, j)$. The required coverage is achieved when $\mathbf{D} \leq \mathbf{C}$.

A CPPIED problem instance is defined as a tuple $\left(\mathcal{T}, \mathbf{O}, \mathbf{D}, p^{\text {scan }},\left(i^{\text {init }}, j^{\text {init }}\right)\right)$ where $\left(i^{\text {init }}, j^{\text {init }}\right)$ is the initial location cell of the robot.

\section{A Hybrid Algorithm Based on DynamiC PROGRAMMING AND THE TRAVELING SALESMAN}

The goal of the proposed algorithm is to define, in lexicographic order, a feasible shortest path (the first objective), consisting of segments, that also minimizes the number of turns (the second objective). A segment is a set of horizontally or vertically adjacent cells that does not contain any turns. The algorithm is composed of two main phases. In the first phase, we construct a set $\mathcal{S}$ of disconnected segments such that a robot traveling along all these segments will achieve the required coverage $D$. In the second phase, we use a $T S P$ reduction to optimally connect the segments obtained in phase 1 and form the desired path $P$. This is similar to the $T S P$ formulation used in [2] to connect paths in sub-areas of a harbor environment. Our algorithm proceeds as follow:

1) Initialize the robot's path $P$ to an empty path, the set of segments $\mathcal{S}$ used to construct path $P$ to the empty set, and the current coverage matrix $\mathbf{C}$ to the null matrix. At each iteration, construct and choose a set of horizontal or a set of vertical segments that maximizes coverage gains (rewards) $\left(\mathcal{H}^{*}\right.$ or $\left.\mathcal{V}^{*}\right)$ and add it to $\mathcal{S}$ (Section IV-A). Iterate until the required coverage is attained by the segments of $\mathcal{S}$, i.e., $\mathbf{D} \leq \mathbf{C}$.

2) Link the segments of $\mathcal{S}$ in an optimal fashion to obtain a feasible path $P$ of minimal length (and minimal number of turns) (Section IV-B).

The aim in phase 1 is to construct a segment set $\mathcal{S}$ allowing the robot to achieve the required coverage with the shortest possible path. Therefore, we need short segments. Intuitively, a set $\mathcal{S}$ with a small cardinality is desirable as well since it will lead to a lower number of turns in phase 2 .

\section{A. Greedily Choosing a Set of Segments $\mathcal{S}$}

Some difficulties in constructing a segment set $\mathcal{S}$ arise due to the extended sensor's range since multiple detections in a given cell may arise from two different segments or more. To overcome this difficulty, we impose, at each iteration, a $2 r$ spacing constraint within the set $\mathcal{H}^{*}$ of horizontal segments and the set $\mathcal{V}^{*}$ of vertical segments. Therefore, the rewards of the parallel segments are independent, which allows us to use polynomial time dynamic programming algorithms to compute both the reward of a robot traveling on a segment, and the optimal sets of segments $\mathcal{H}^{*}$ and $\mathcal{V}^{*}$.
In order to compute the horizontal gain in cell $(i, j), \mathbf{H}_{i j}$, let $p^{\mathrm{C}}(i, j, k)$ be the updated probability of detection when a cell $(i, j)$ is scanned from a distance of $k$ cells:

$$
p^{\mathrm{C}}(i, j, k)=\mathbf{C}_{i j}+\left(1-\mathbf{C}_{i j}\right) p^{\mathrm{scan}}\left(k, \mathbf{O}_{i j}\right) .
$$

The gain obtained by scanning cell $(i, j)$ from distance $k$ can be defined as:

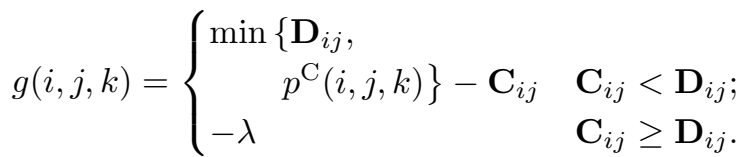

If cell $(i, j)$ is not already covered, i.e., $\mathbf{C}_{i j}<\mathbf{D}_{i j}$, the gain equals the increment in probability of detection in $(i, j)$ up to the required probability of detection value, i.e., $\min \left\{\mathbf{D}_{i j}, p^{\mathrm{C}}(i, j, k)\right\}-\mathbf{C}_{i j}$. Otherwise, cell $(i, j)$ is covered, i.e., $\mathbf{C}_{i j} \geq \mathbf{D}_{i j}$, and we impose a small penalty $\lambda$ for overcoverage. Let $G(i, j)$ be the sum of the gains, in probability of detection, in the cells within the range of the robot:

$$
G(i, j)=\sum_{k=1}^{r} g(i-k+1, j, k)+\sum_{k=1}^{r} g(i+k, j, k) .
$$

Since we wish to find the shortest possible segment with the highest coverage, the segment ends before the first cell which gain $G(i, j)$ is less than or equal to zero. Therefore, the horizontal gain of a cell $(i, j), \mathbf{H}_{i j}$, is defined as follows:

$$
\mathbf{H}_{i j}= \begin{cases}G(i, j) & G(i, j)>0 \\ -\infty & G(i, j) \leq 0\end{cases}
$$

The endpoints $a$ and $b$ that define the segment $h_{i}$ such that the sum of its gains is maximal are identified for each row $i$ as follows:

$$
K\left(h_{i}\right)=\max _{a, b} \sum_{j=a}^{b} \mathbf{H}_{i j} .
$$

This problem, called the Maximum subarray problem, is solvable in linear time using Kadane's algorithm [23], a simple example of dynamic programming.

The optimal horizontal set $\mathcal{H}^{*}$ is the subset of segments that maximizes the sum of the horizontal gains over the rows subject to a $2 r$ spacing constraint:

$$
\max _{I \subset\{1 . . m\}\left|i, i^{\prime} \in I \Rightarrow\right| i-i^{\prime} \mid \geq 2 r} \sum_{i \in I} K\left(h_{i}\right) .
$$

We compute $\mathcal{H}^{*}$ using the following recurrence relation:

$$
\mathbf{h}_{i}^{*}= \begin{cases}K\left(h_{i}\right) & 1 \leq i \leq 2 r ; \\ \max \left\{\mathbf{h}^{*}{ }_{i-1},\right. & 2 r<i \leq m, \\ \left.K\left(h_{i}\right)+\mathbf{h}^{*}{ }_{i-2 r}\right\} & \end{cases}
$$

where $\mathbf{h}^{*}{ }_{i}$ is the maximum gain achieved by a robot when it travels along segment $h_{i}$. With this technique, choosing an optimal set of segments under a $2 r$ spacing constraint is done in polynomial time. The set $\mathcal{V}^{*}$ is computed in the same fashion following a map rotation of $90^{\circ}$. The set among $\mathcal{H}^{*}$ or $\mathcal{V}^{*}$ providing the highest coverage is added to $\mathcal{S}$. Ties are broken using the set with the smallest cardinality. Note that there is no spacing constraint in $\mathcal{S}$. 


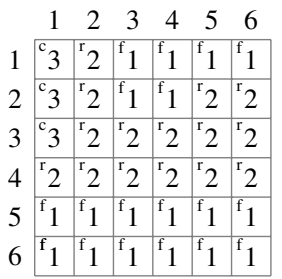

(a) Initialization: $\mathcal{S}$ is empty; labels show the seabed type and the number of scans left.

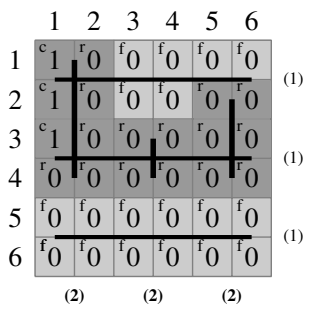

(c) Iteration 2: a vertical segment set $\mathcal{V}^{*}$ (labeled (2)) is added to $\mathcal{S}$.

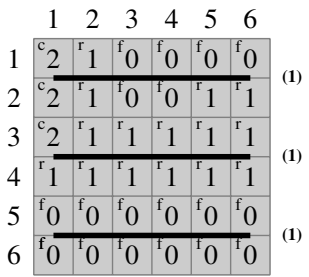

(b) Iteration 1: a horizontal segment set $\mathcal{H}^{*}$ (labeled (1)), is added to $\mathcal{S}$.

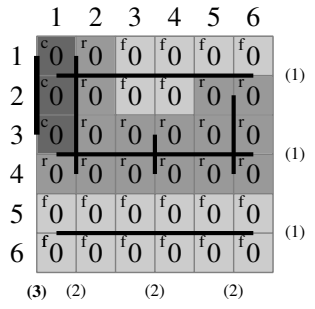

(d) Iteration 3: a vertical segment set $\mathcal{V}^{*}$ (labeled (3)) is added to $\mathcal{S}$.
Fig. 3. The construction of the segment set $\mathcal{S}$ on a map with $\mathcal{T}=$ \{flat (f), ripples (r), complex (c) $\}$, and $r=1$; dark gray shaded cells were scanned three times, medium gray shaded cells were scanned twice, light gray shaded cells were scanned once.

1) Example: Fig. 3 presents an example for choosing an optimal segment set on a $6 \times 6$ grid with $r=1$. In each cell of the grid, we indicate the number of robot scans needed to achieve the required coverage $\mathbf{D}$. This number is an upper bound that depends on the seabed type, on the current coverage matrix $\mathbf{C}$, and on the probability of detection $p^{\text {scan }}$. The set $\mathcal{S}$ is initialized to the empty set (Fig. 3a), and $\mathbf{C}=0$. At iteration 1 (Fig. 3b), the procedure generates a first set $\mathcal{H}^{*}$ of horizontal segments and a first set $\mathcal{V}^{*}$ of vertical segments. $\mathcal{H}^{*}$ is then added to $\mathcal{S}\left(\mathcal{V}^{*}\right.$ is equivalent in terms of gain, in this case). At iteration 2 (Fig. 3c), the updated current coverage matrix $\mathbf{C}$ is used to compute two new sets $\mathcal{H}^{*}$ and $\mathcal{V}^{*}$. The algorithm adds $\mathcal{V}^{*}$ to $\mathcal{S}$ since it achieves a higher gain. At iteration 3 (Fig. 3d), the algorithm chooses $\mathcal{V}^{*}$ (a single segment) and adds it to $\mathcal{S}$.

\section{B. Reducing to a TSP and Reconstructing the Path P}

In this phase, a connected path $P$ including all the segments in $\mathcal{S}$ is constructed using a $T S P$ reduction. Let $\mathcal{V}(G)$ be the set of nodes of graph $G, \mathcal{E}(G)$ be its set of edges, and $c(e)$ be a cost function on $\mathcal{E}(G)$. The goal is to find a cycle of minimal cost that visits each node once.

For each segment $s_{i}$ in $\mathcal{S}$ with endpoints $u_{i}$ and $w_{i}$, we create three nodes in $\mathcal{V}(G): u_{i}, w_{i}$, and $v_{i}$ and two edges $\left(u_{i}, v_{i}\right)$ and $\left(v_{i}, w_{i}\right)$. The node $v_{i}$ is a dummy node used to force the algorithm to travel over the segment between $u_{i}$ and $w_{i}$. The cost of these two edges is null. For every pair of segments $s_{i}$ and $s_{j}$ in $\mathcal{S}$, we create four edges: $\left(u_{i}, u_{j}\right)$, $\left(u_{i}, w_{j}\right),\left(w_{i}, u_{j}\right)$, and $\left(w_{i}, w_{j}\right)$. The cost of these edges corresponds to the shortest distance in the number of robot's moves needed to connect the endpoints. Finally, let $u^{s}$ be

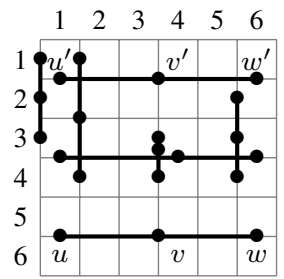

(a) Step 1: generate the

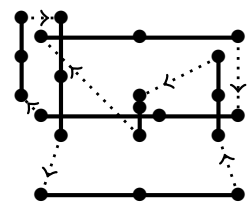

(c) Step 3: find an optimal cycle (dotted lines) graph's nodes.

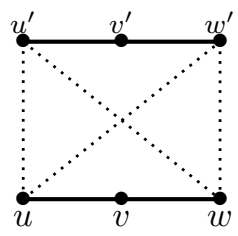

(b) Step 2: generate the

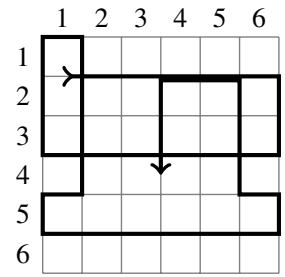

(d) Step 4: reconstruct the path. graph's edges (dotted lines).

Fig. 4. The TSP reduction and the path reconstruction on the segments set $\mathcal{S}$ of Fig. 3 .

the starting position of the robot. We create a source node $u^{s}$ connected to all endpoints of the segments with distances given by the shortest distance in number of moves. We create a sink node $w^{s}$ connected to all segments' endpoints with a null distance. We create a dummy node $v^{s}$ that is connected to $u^{s}$ and $w^{s}$ by two edges of null cost.

Solving the TSP consists of finding a cycle of minimum cost that passes through each node exactly once. Note that such a cycle would have to visit each node $v_{i}$ once. Since this node is only connected to the two endpoints $u_{i}, w_{i}$, it forces the cycle to enter by one endpoint of the segment and to leave by the other endpoint. The same principle applies for the node $v^{s}$ that is only connected to the source node $u^{s}$ and the sink node $w^{s}$. Therefore, a cycle starts at node $w^{s}$, visits all the segments, and returns to $w^{s}$ through $v^{s}$. Removing the node $w^{s}$ and $v^{s}$ from the cycle yields the solution path $P$ we are looking for. To find the lowest cost cycle, and therefore the solution path, we chose the Concorde solver [24].

1) Example: Fig. 4 shows a $T S P$ reduction and a path reconstruction example on the segments set $\mathcal{S}$ of Fig. 3. First, the reduction process generates the graph nodes represented by dots on Fig. 4a. The robot starts in cell $(1,1)$. Therefore, the source node $u^{s}$ is positioned in $(1,1)$, the sink node $w^{s}$ and the dummy node $v^{s}$ are positioned at the same place. Second, it links the segments endpoints by creating edges. Fig. 4b shows the edges added to the graph $G$ for segments $s=(u, v, w)$ and $s^{\prime}=\left(u^{\prime}, v^{\prime}, w^{\prime}\right)$. Third, on Fig. 4c, the Concorde solver finds an optimal cycle that starts from the source and goes through all the nodes before returning to the source node $u^{s}$ by the sink node $w^{s}$ then the dummy node $v^{s}$. Finally, on Fig. 4d, the robot's path is reconstructed. As shown, there are no links between the last robot's position $(4,3)$ and its initial position in $(1,1)$ : We simply ignore the sink and the dummy nodes during path reconstruction. 
TABLE I

PROBLEM INSTANCES PUBLISHED IN [7]

\begin{tabular}{|c|c|c|c|}
\hline No & $\begin{array}{c}\text { Ocean bed } \\
\mathbf{O}\end{array}$ & $\begin{array}{l}\text { Required coverage } \\
\mathbf{D}_{i j}(\forall i, j) \\
\end{array}$ & $\begin{array}{c}\text { Detection prob. } \\
p^{\text {scan }}\end{array}$ \\
\hline 1 & [7, Fig. 5.1] & 0.9 & {$\left[\begin{array}{ccc}0.6 & 0.65 & 0.62 \\
0.7 & 0.85 & 0.8 \\
0.99 & 0.99 & 0.99\end{array}\right]$} \\
\hline 2 & [7, Fig. 5.4] & 0.9 & {$\left[\begin{array}{ccc}0.7 & 0.8 & 0.75 \\
0.99 & 0.99 & 0.99 \\
0.91 & 0.95 & 0.92\end{array}\right]$} \\
\hline 3 & [7, Fig. 5.8] & 0.9 & $\begin{array}{l}{\left[\begin{array}{ccc}0.8 & 0.8 & 0.8 \\
0.8 & 0.8 & 0.8 \\
0.91 & 0.91 & 0.91\end{array}\right]} \\
{\left[\begin{array}{lll}0.51 & 0.51 & 0.51\end{array}\right]}\end{array}$ \\
\hline 4 & [7, Fig. 5.11] & 0.75 & $\begin{array}{l}{\left[\begin{array}{ccc}0.8 & 0.8 & 0.8 \\
0.91 & 0.91 & 0.91\end{array}\right]} \\
{\left[\begin{array}{lll}0.51 & 0.51 & 0.51\end{array}\right]}\end{array}$ \\
\hline 5 & [7, Fig. 5.14] & 0.75 & {$\left[\begin{array}{ccc}0.8 & 0.8 & 0.8 \\
0.99 & 0.99 & 0.99\end{array}\right]$} \\
\hline 6 & [7, Fig. 5.16] & 0.9 & {$\left[\begin{array}{ccc}0.6 & 0.6 & 0.6 \\
0.8 & 0.8 & 0.8 \\
0.91 & 0.91 & 0.91\end{array}\right]$} \\
\hline 7 & [7, Fig. 5.18] & 0.85 & {$\left[\begin{array}{ccc}0.6 & 0.6 & 0.6 \\
0.8 & 0.8 & 0.8 \\
0.91 & 0.91 & 0.91\end{array}\right]$} \\
\hline
\end{tabular}

\section{EXPERIMENTATION}

We present the results of our DpSweeper algorithm, implemented in $\mathrm{C}++$, obtained on an Intel(R) Core(TM) i7-Q740 CPU with 8 GB of RAM. A comparison with the results of the HCPP algorithm on seven instances published in [7] is included. Table I summarizes the instances in increasing order of complexity. The robot starts in the top left corner of the grid. The required coverage matrix $\mathbf{D}$ is uniform, i.e., all cells have the same required coverage probability. We use a range $r=3$. The seabed maps $\mathbf{O}$, containing more than 21000 cells, can consist of three different seabed types: flat (f), ripples (r), and complex (c) seabed. The first instance [7, Fig. 5.1] has a flat seabed: a lawnmower pattern is sufficient to cover it. The second [7, Fig. 5.4] is made of a flat seabed with a rectangular complex seabed patch in the middle. The third [7, Fig. 5.8] has three complex seabed patches. The fourth [7, Fig. 5.11] has a circle of complex seabed in the middle. The fifth [7, Fig. 5.14] has a fragmented circle of complex seabed in the middle. The sixth [7, Fig. 5.16] is a real map containing three different seabed types. The seventh [7, Fig. 5.18] is a realistic randomly generated map containing three different seabed types.

Table II presents the results of both the HCPP and the DpSweeper algorithms. As in [7], we use the following lexicographic order of criteria: First minimize path length, then the number of turns. The required coverage is attained in all cases. We see that the DpSweeper algorithm outperforms the HCPP algorithm on the first criterion in all instances except for instances 2 and 5. Following a close inspection of the figures published in [7], we noticed that on the spot $180^{\circ}$ turns were allowed. Our more restrictive assumptions (not allowing on the spot $180^{\circ}$ turns), although not a DpSweeper limitation, are closer to the physical constraints of AUVs and actually favor HCPP. This may explain the slightly longer
TABLE II

COMPARISON OF DPSWEEPER TO HCPP [7]

\begin{tabular}{c|cc|ccccc}
\hline & \multicolumn{2}{|c|}{$\boldsymbol{H C P P}^{\dagger}$} & \multicolumn{5}{c}{ DpSweeper } \\
\hline & \multicolumn{2}{|c|}{ Solution } & \multicolumn{2}{c}{ Solution } & & TSP & Time (s) \\
No & Length & Turns & Length & Turns & nodes & $T S P$ & Total \\
\hline 1 & 3777 & 40 & $\mathbf{3 7 5 5}$ & 41 & 66 & $<1$ & $<1$ \\
2 & $\mathbf{4 5 9 9}$ & $\mathbf{6 0}$ & 4628 & 61 & 99 & $<1$ & 1 \\
3 & 5556 & 82 & $\mathbf{5 3 2 1}$ & 95 & 144 & $<1$ & 1 \\
4 & 5494 & 77 & $\mathbf{5 3 6 3}$ & $\mathbf{7 5}$ & 117 & $<1$ & 1 \\
5 & $\mathbf{5 1 1 9}$ & 114 & 5151 & 107 & 162 & 1 & 2 \\
6 & 6689 & 136 & $\mathbf{5 6 8 1}$ & 270 & 402 & 45 & 52 \\
7 & 7141 & 137 & $\mathbf{5 7 3 1}$ & 174 & 273 & 12 & 15 \\
\hline
\end{tabular}

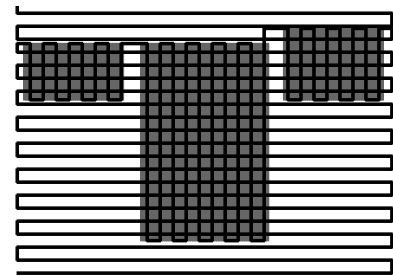

(a) Solution to problem 3

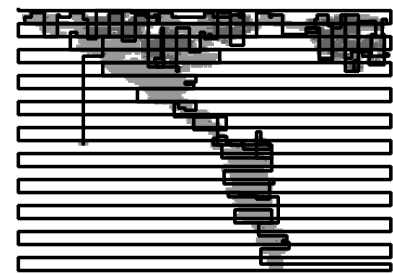

(c) Solution to problem 6

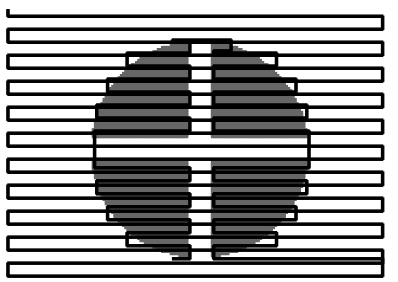

(b) Solution to problem 5

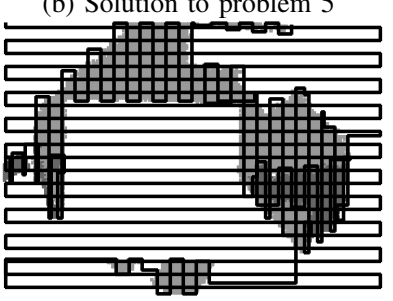

(d) Solution to problem 7
Fig. 5. Solutions found by DpSweeper; complex, ripples, and flat seabed cells are respectively filled with dark gray, gray, and white.

path we found on instances 2 and 5. For the instances 6 and 7, our solution has significantly more turns than HCPP. On these instances, generating more turns was necessary to diminish the path length of respectively more than $15 \%$ and $20 \%$ when compared to [7], which is coherent with the lexicographic order of the minimization objectives.

The DpSweeper algorithm solved instances 1 to 4 in one second or less and instance 5 in two seconds. The solving times of the most realistic instances (6 and 7) are within a minute. These times include both the segment generation process and the Concorde solver calls. We chose to use Concorde instead of a TSP heuristic since it is the state of the art: It solves most TSPLIB [25] instances (sometimes with more than 2000 nodes) within a few minutes. Although Concorde may consume more time than a heuristic, it finds the optimal tour to all our instances within a few seconds.

Fig. 5 shows some of the solutions provided by our algorithm. On the problem 3 (Fig. 5a), we see the tendency of the algorithm to generate a simple lawnmower pattern that minimizes both the path length and the number of turns. It starts with a horizontal lawnmower pattern. Then, on the third row, it follows a vertical lawnmower to cover the complex seabed patches. Finally, it comes back to its 
horizontal lawnmower pattern to cover all the grid. We notice a similar behavior on problem 5 (Fig. 5b). The seabed of this grid forces the algorithm to adapt the length of each segment to follow the fragmented circle edges. For problem 6 (Fig. 5c), the algorithm first plans a complete lawnmower pattern. Then, it goes up towards and over the closest ripples seabed and the complex patches. Finally, it passes over complex seabed patches again to achieve the required coverage, and then aims for the furthest ripples patches. It ends its course on the left hand side of the map. A similar behavior occurs in Fig. 5d.

In addition to its superior results, the main advantage DpSweeper over HCPP is that it is general, and contrary to HCPP, does not require lengthy, instance specific, finetuning. Furthermore, its very short computational time makes it possible to obtain a high quality path rapidly, an important characteristic for algorithms in practical contexts.

\section{CONCLUSION}

We have presented a novel algorithm (DpSweeper) for coverage path planning using imperfect sensors with an extended detection range $(C P P I E D)$. In order to obtain shortest feasible paths with small numbers of turns, the algorithm consists of two main phases: (1) greedily constructing a partial path made of segments to guarantee that the required coverage is achieved (dynamic programming); (2) optimally linking segments to create a path that is within the robot's physical constraints ( TSP reduction). The algorithm yields favorable results in a very short time compared to the literature. It is flexible and can be applied to general complex seabed environments. In contrast with the only other algorithm that tackles the CPPIED problem, it does not require customized fine-tuning for individual environments. Even though the TSP problem is $\mathcal{N} \mathcal{P}$-hard [26], the instances resulting from our real and practical CPPIED problem instances are within the reach of the Concorde TSP solver that we used. TSP reductions are found in the literature to solve part of decomposed coverage problems (e.g., [2]) or other similar problems (e.g., [27]). However, most of them use the TSP to find a sequence of large regions where they assume a fixed coverage pattern. We use the TSP directly to order a sequence of path components (segments) on the whole environment. Our algorithm is not limited to an underwater context and may be used in other obstacle-free environments.

\section{ACKNOWLEDGMENT}

We wish to thank P. Giguère for his insightful suggestions, and the Ocean Systems Lab for providing us with the problem instances found in [7].

\section{REFERENCES}

[1] J. Nicholson and A. Healey, "The present state of autonomous underwater vehicle (AUV) applications and technologies," Marine Technology Society Journal, vol. 42, no. 1, pp. 44-51, 2008.

[2] C. Fang and S. Anstee, "Coverage path planning for harbour seabed surveys using an autonomous underwater vehicle," in Proc. of OCEANS 2010 IEEE-Sydney, Sydney, Australia, May 2010, pp. 18.
[3] S. Reed, Y. Petillot, and J. Bell, "An automatic approach to the detection and extraction of mine features in sidescan sonar," IEEE J. Oceanic Eng., vol. 28, no. 1, pp. 90-105, 2003.

[4] D. W. Gage, "Randomized search strategies with imperfect sensors," in Proc. of SPIE Mobile Robots VIII, San Diego, CA, Sept. 1993, pp. 270-279.

[5] —-, "Many-robot MCM search systems," in Proc. of Autonomous Vehicles in Mine Countermeasures Symposium, A. Bottoms, J. Eagle, and H. Bayless, Eds., Monterey, CA, Apr. 1995.

[6] R. Mannadiar and I. Rekleitis, "Optimal coverage of a known arbitrary environment," in Proc. of IEEE International Conference on Robotics and Automation (ICRA'10), Anchorage, AK, May 2010, pp. 55255530.

[7] M. Drabovich, "Automated mission trajectory planning for mine countermeasures operations," Master's thesis, Heriot Watt University, Edinburgh, Scotland, UK, 2008.

[8] D. P. Williams, "On optimal AUV track-spacing for underwater mine detection," in Proc. of IEEE International Conference on Robotics and Automation (ICRA'10), Anchorage, AK, May 2010, pp. 4755-4762.

[9] J. R. Stack and C. M. Smith, "Combining random and data-driven coverage planning for underwater mine detection," in Proc. of OCEANS 2003 MTS/IEEE, vol. 5, San Diego, CA, Sept. 2003, pp. 2463-2468.

[10] T. Oksanen and A. Visala, "Coverage path planning algorithms for agricultural field machines," Journal of Field Robotics, vol. 26, no. 8, pp. 651-668, 2009.

[11] E. Galceran and M. Carreras, "Coverage path planning for marine habitat mapping," in Proc. of OCEANS 2012 MTS/IEEE, Hampton Roads, VA, USA, Oct. 2012, pp. 1-8.

[12] R. N. De Carvalho, H. Vidal, P. Vieira, and M. Ribeiro, "Complete coverage path planning and guidance for cleaning robots," in Proc. of IEEE International Symposium on Industrial Electronics (ISIE'97), vol. 2, Guimarães, Portugal, July 1997, pp. 677-682.

[13] M. Morin, L. Lamontagne, I. Abi-Zeid, P. Lang, and P. Maupin, "The optimal searcher path problem with a visibility criterion in discrete time and space," in Proc. of the 12th International Conference on Information Fusion (FUSION'09). Seattle, WA: ISIF IEEE, July 2009, pp. 2217-2224.

[14] I. Abi-Zeid and J. R. Frost, "Sarplan: A decision support system for canadian search and rescue operations," European Journal of Operational Research, vol. 162, no. 3, pp. 630-653, 2005.

[15] B. Nguyen and D. Hopkin, "Modeling autonomous underwater vehicle (AUV) operations in mine hunting," in OCEANS 2005 IEEE-Europe, vol. 1, Brest, France, June 2005, pp. 533-538.

[16] E. Acar, Y. Zhang, H. Choset, M. Schervish, A. G. Costa, R. Melamud, D. Lean, and A. Graveline, "Path planning for robotic demining and development of a test platform," in Proc. of the 8th International Conference on Field and Service Robotics (FSR 2012), Matsushima, Japan, July 2001, pp. 161-168.

[17] Y. Zhang, M. Schervish, E. U. Acar, and H. Choset, "Probabilistic methods for robotic landmine search," in Proc. of the IEEE/RSJ International Conference on Intelligent Robots and Systems (IROS'01), vol. 3, Maui, Hawaii, Oct./Nov. 2001, pp. 1525-1532.

[18] H. Choset, "Coverage for robotics," Annals of Mathematics and Artificial Intelligence, vol. 31, no. 1-4, pp. 113-126, 2001.

[19] S. Russel and P. Norvig, Intelligence artificielle, 2nd ed. Paris, France: Pearson Education France, 2006.

[20] A. Elfes, "Using occupancy grids for mobile robot perception and navigation," IEEE Computer, vol. 22, no. 6, pp. 46-57, 1989.

[21] L. Paull, S. Saeedi, and H. Li, "Path planning for autonomous underwater vehicles," in Robot Autonomy. Springer, 2013, pp. 177223.

[22] G. Cannata and A. Sgorbissa, "A minimalist algorithm for multirobot continuous coverage," IEEE Transactions on Robotics, vol. 27, no. 2, pp. 297-312, 2011.

[23] J. Bentley, "Programming pearls: algorithm design techniques," Communications of the ACM, vol. 27, no. 9, pp. 865-873, 1984.

[24] D. Applegate, R. Bixby, V. Chvatal, and W. Cook. Concorde TSP solver. [Online]. Available: http://www.tsp.gatech.edu/concorde

[25] G. Reinelt, "TSPLIB-A traveling salesman problem library," ORSA Journal on Computing, vol. 3, no. 4, pp. 376-384, 1991.

[26] M. R. Garey and D. S. Johnson, Computers and intractability. San Francisco, CA: Freeman, 1979, vol. 174.

[27] S. Ntafos, "Watchman routes under limited visibility," Computational Geometry, vol. 1, no. 3, pp. 149-170, 1992. 